\title{
Correction to "Oral Calcium Supplements Do Not Affect the Progression of Aortic Valve Calcification or Coronary Artery Calcification"
}

In the above mentioned article, ${ }^{1}$ Figure 2 was published incorrectly. The correct version of the figure appears below. The electronic version on the Journal's website has been corrected. We apologize for the error, and we regret any confusion or inconvenience it may have caused.

(doi: 10.3122/jabfm.2012.02.120009.)

Figure 2. Comparison of the change in coronary artery calcification (CAC) from baseline to follow-up (vertical lines show SD) in women who took calcium supplementation and those that did not take supplementation. $P$ is comparing rates of change in coronary artery calcium score (supplement vs no supplement). Data source: the Epidemiology of Coronary Artery Calcification (ECAC) study. ${ }^{12}$

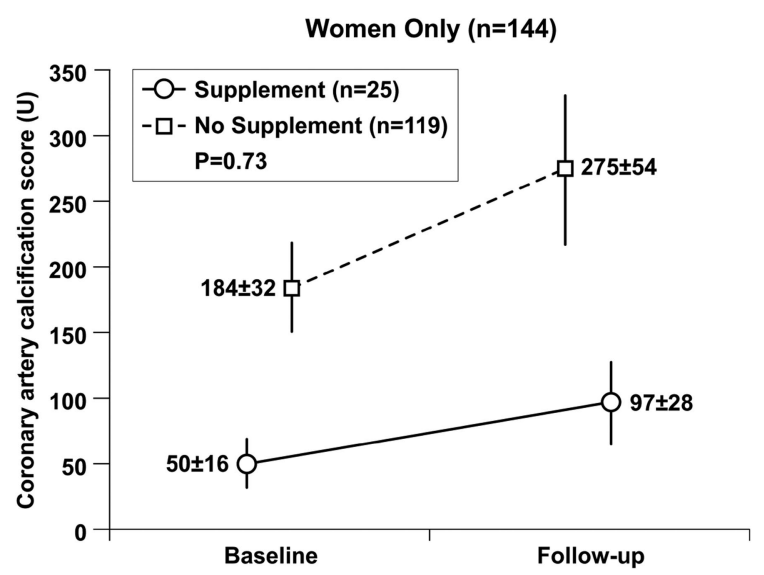

\section{Reference}

1. Bhakta M, Bruce C, Messika-Zeitoun D, et al. Oral calcium supplements do not affect the progression of aortic valve calcification or coronary artery calcification. J Am Board Fam Med 2009;22:610-6. 